\title{
Teleportation Systems Toward a Quantum Internet
}

\author{
Raju Valivarthi®,$^{1,2}$ Samantha I. Davis, ${ }^{1,2}$ Cristián Peña, ${ }^{1,2,3}$ Si Xie $\odot,{ }^{1,2}$ Nikolai Lauk, ${ }^{1,2}$ \\ Lautaro Narváez $\odot,{ }^{1,2}$ Jason P. Allmaras $\odot,{ }^{4}$ Andrew D. Beyer, ${ }^{4}$ Yewon Gim, ${ }^{2,5}$ Meraj Hussein, ${ }^{2}$ \\ George Iskander $\odot,{ }^{1}$ Hyunseong Linus Kim $\odot,{ }^{1,2}$ Boris Korzh $\odot,{ }^{4}$ Andrew Mueller, ${ }^{1}$ Mandy Rominsky, ${ }^{3}$ \\ Matthew Shaw, ${ }^{4}$ Dawn Tang $\circledast,{ }^{1,2}$ Emma E. Wollman, ${ }^{4}$ Christoph Simon, ${ }^{6}$ Panagiotis Spentzouris, ${ }^{3}$ \\ Daniel Oblak, ${ }^{6}$ Neil Sinclair, ${ }^{1,2,7}$ and Maria Spiropulu ${ }^{1,2, *}$ \\ ${ }^{1}$ Division of Physics, Mathematics and Astronomy, California Institute of Technology, Pasadena, \\ California 91125, USA \\ ${ }^{2}$ Alliance for Quantum Technologies (AQT), California Institute of Technology, Pasadena, California 91125, USA \\ ${ }^{3}$ Fermi National Accelerator Laboratory, Batavia, Illinois 60510, USA \\ ${ }^{4}$ Jet Propulsion Laboratory, California Institute of Technology, Pasadena, California 91109, USA \\ ${ }^{5}$ AT\& T Foundry, Palo Alto, California 94301, USA \\ ${ }^{6}$ Institute for Quantum Science and Technology, and Department of Physics and Astronomy, University of Calgary, \\ Calgary, Alberta T2N 1N4, Canada \\ ${ }^{7}$ John A. Paulson School of Engineering and Applied Sciences, Harvard University, Cambridge, \\ Massachusetts 02138, USA
}

(Received 28 July 2020; accepted 16 October 2020; published 4 December 2020; corrected 22 July 2021)

Quantum teleportation is essential for many quantum information technologies, including long-distance quantum networks. Using fiber-coupled devices, including state-of-the-art low-noise superconducting nanowire single-photon detectors and off-the-shelf optics, we achieve conditional quantum teleportation of time-bin qubits at the telecommunication wavelength of $1536.5 \mathrm{~nm}$. We measure teleportation fidelities of $\geq 90 \%$ that are consistent with an analytical model of our system, which includes realistic imperfections. To demonstrate the compatibility of our setup with deployed quantum networks, we teleport qubits over $22 \mathrm{~km}$ of single-mode fiber while transmitting qubits over an additional $22 \mathrm{~km}$ of fiber. Our systems, which are compatible with emerging solid-state quantum devices, provide a realistic foundation for a high-fidelity quantum Internet with practical devices.

DOI: 10.1103/PRXQuantum.1.020317

\section{INTRODUCTION}

Quantum teleportation [1], one of the most captivating predictions of quantum theory, has been widely investigated since its seminal demonstrations over 20 years ago [2-4]. This is due to its connections to fundamental physics [5-14] and its central role in the realization of quantum information technology such as quantum computers and networks [15-19]. The goal of a quantum network is to distribute qubits between different locations, a key task for quantum cryptography, distributed quantum computing, and sensing. A quantum network is expected to form part of a future quantum Internet [20-22]: a globally distributed

*smaria@caltech.edu

Published by the American Physical Society under the terms of the Creative Commons Attribution 4.0 International license. Further distribution of this work must maintain attribution to the author(s) and the published article's title, journal citation, and DOI. set of quantum processors, sensors, or users thereof that are mutually connected over a network capable of allocating quantum resources (e.g., qubits and entangled states) between locations. Many architectures for quantum networks require quantum teleportation, such as star-type networks that distribute entanglement from a central location or quantum repeaters that overcome the rate-loss trade-off of direct transmission of qubits [19,23-26].

Quantum teleportation of a qubit can be achieved by performing a Bell-state measurement (BSM) between the qubit and another that forms one member of an entangled Bell state $[1,18,27]$. The quality of the teleportation is often characterized by the fidelity $F=\langle\psi|\rho| \psi\rangle$ of the teleported state $\rho$ with respect to the state $|\psi\rangle$ accomplished by ideal generation and teleportation [15]. This metric is becoming increasingly important as quantum networks move beyond specific applications, such as quantum key distribution, and toward the quantum Internet.

Polarization qubits have been preferred for demonstrations of quantum teleportation over free-space channels 
[28-30], including the recent ground-to-satellite quantum teleportation [31], because of their ease of preparation and measurement, as well as the lack of polarization rotation in free space. Qubits encoded by the time of arrival of individual photons, i.e., time-bin qubits [32], are useful for fiber networks due to their simplicity of generation, interfacing with quantum devices, as well as the independence of dynamic polarization transformations of realworld fibers. Individual telecom-band photons (around $1.5 \mu \mathrm{m}$ wavelength) are ideal carriers of qubits in networks due to their ability to rapidly travel over long distances in deployed optical fibers [17,33-35] or atmospheric channels [36], among other properties. Moreover, the improvement and growing availability of sources and detectors of individual telecom-band photons has accelerated progress toward workable quantum networks and associated technologies, such as quantum memories [37], transducers $[38,39]$, or quantum nondestructive measurement devices [40].

Teleportation of telecom-band photonic time-bin qubits has been performed inside and outside the laboratory, with impressive results [33-35,41-46]. Despite this, there has been little work to increase $F$ beyond approximately $90 \%$ for these qubits, in particular using practical devices that allow straightforward replication and deployment of quantum networks (e.g., using fiber-coupled and commercially available devices). Moreover, it is desirable to develop teleportation systems that are forward compatible with emerging quantum devices for the quantum Internet.

In the context of the California Institute of Technology (Caltech) multidisciplinary multi-institutional collaborative public-private research program on Intelligent Quantum Networks and Technologies (IN-Q-NET) founded in 2017 with AT\&T as well as the Fermi National Accelerator Laboratory and the Jet Propulsion Laboratory, we have designed, built, commissioned, and deployed two quantum teleportation systems: one at Fermilab, the Fermilab Quantum Network (FQNET), and one at Caltech's Lauritsen Laboratory for High Energy Physics, the Caltech Quantum Network (CQNET). The CQNET system serves as a research and development (R\&D), prototyping, and commissioning system, while FQNET serves as an expandable system, for scaling up to long distances, and is used in multiple projects funded currently by the United States (U.S.) Department of Energy's Office of High Energy Physics (HEP) and Advanced Scientific Research Computing (ASCR). The material- and devices-level R\&D of both systems is facilitated and funded by the Office of Basic Energy Sciences (BES). Both systems are accessible to quantum researchers for R\&D purposes as well as testing and integration of various novel devices, such as, for example, on-chip integrated nanophotonic devices and quantum memories, needed to upgrade such systems toward a realistic quantum Internet. Importantly, both systems are also used for improvements of the entanglement quality and distribution, with emphasis on implementation of protocols with complex entangled states toward advanced and complex quantum communications channels. These will assist in studies of systems that implement new teleportation protocols the gravitational duals of which correspond to wormholes [47], error-correlation properties of wormhole teleportation, and on-chip codes as well as possible implementation of protocols on quantum optics communication platforms. Hence the systems serve both fundamental quantum information science as well as quantum technologies.

Here, we perform quantum teleportation of time-bin qubits, conditioned on a successful BSM, at a wavelength of $1536.5 \mathrm{~nm}$ with an average $F \geq 90 \%$. This is accomplished using a compact setup of fiber-coupled devices, including low-dark-count single-photon detectors and offthe-shelf optics, allowing straightforward reproduction for multinode networks. To illustrate network compatibility, teleportation is performed with up to $44 \mathrm{~km}$ of singlemode fiber between the qubit generation and the measurement of the teleported qubit, and is facilitated using semiautonomous control, monitoring, and synchronization systems, with results collected using scalable acquisition hardware. Our system, which operates at a clock rate of 90 $\mathrm{MHz}$, can be run remotely for several days without interruption and can yield teleportation rates of a few Hertz using the full length of fiber. Our qubits are also compatible with erbium-doped crystals, e.g., $\mathrm{Er}: \mathrm{Y}_{2} \mathrm{SiO}_{5}$, which are used to develop quantum network devices such as memories and transducers [48-50]. The 1536.5-nm operating wavelength is within the low-loss ( $C$-band) telecommunication window for long-haul communication and where a variety of off-the-shelf equipment is available. Finally, we develop an analytical model of our system, which includes experimental imperfections, predicting that the fidelity can be improved further toward unity by well-understood methods (such as improvement in photon indistinguishability). Our demonstrations provide a step toward a workable quantum network with practical and replicable nodes, such as the ambitious U.S. Department of Energy quantum research network envisioned to link the U.S. National Laboratories.

In the following, we describe the components of our systems as well as characterization measurements that support our teleportation results, including the fidelity of our entangled Bell state and the Hong-OuMandel (HOM) interference [51] that underpins the success of the BSM. We then present our teleportation results using both quantum state tomography (QST) [52] and projection measurements based on a decoystate method (DSM) [53], followed by a discussion of our model. We conclude by considering improvements toward near-unit fidelity and gigahertz-level teleportation rates. 


\section{SETUP}

Our fiber-based experimental system is summarized in Fig. 1. It allows us to demonstrate a quantum teleportation protocol in which a photonic qubit (provided by Alice) is interfered with one member of an entangled photon pair (from Bob) and projected (by Charlie) onto a Bell state, whereby the state of Alice's qubit can be transferred to the remaining member of Bob's entangled photon pair. Up to 22 (11) $\mathrm{km}$ of single-mode fiber is introduced between Alice and Charlie (Bob and Charlie), as well as up to another $11 \mathrm{~km}$ at Bob, depending on the experiment (see Sec. III). All qubits are generated at the clock rate, with all of their measurements collected using a data acquisition (DAQ) system. Each of the Alice, Bob, and Charlie subsystems are further detailed in the following subsections, with the DAQ subsystem described in Appendix A 1.

\section{A. Alice: single-qubit generation}

To generate the time-bin qubit that Alice will teleport to Bob, light from a fiber-coupled 1536.5 -nm continuouswave $(\mathrm{cw})$ laser is input into a lithium niobate intensity modulator (IM). We drive the IM with one pulse, or two pulses separated by $2 \mathrm{~ns}$. Each pulse is of approximately $65 \mathrm{ps}$ full width at half maximum (FWHM) duration. The pulses are produced by an arbitrary-waveform generator (AWG) and amplified by a 27-dB-gain high-bandwidth amplifier to generate optical pulses that have an extinction ratio of up to $22 \mathrm{~dB}$. We note that this method of creating time-bin qubits offers us flexibility not only in

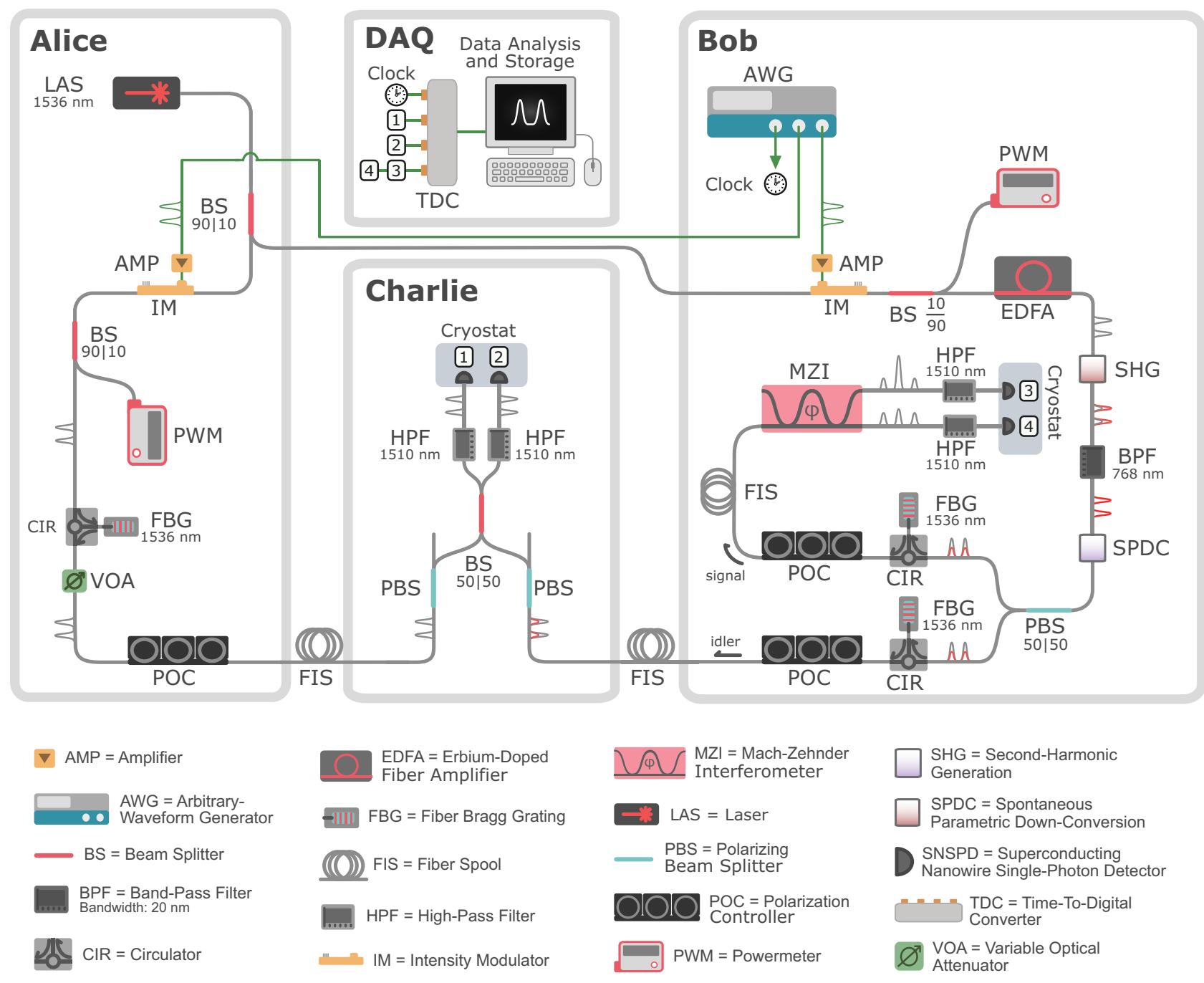

FIG. 1. A schematic diagram of the quantum teleportation system consisting of Alice, Bob, Charlie, and the data acquisition (DAQ) subsystems. See the main text for descriptions of each subsystem. One cryostat is used to house all SNSPDs: it is drawn as two for ease of explanation. The detection signals generated by each of the SNSPDs are labelled 1-4 and collected at the TDC, with 3 and 4 being time multiplexed. All individual components are labeled in the legend, with single-mode optical fibers (electronic cables) in gray (green), and with uni- and bichromatic (i.e., unfiltered) optical pulses indicated. 
terms of choosing a suitable time-bin separation but also for synchronizing qubits originating from different nodes in a network. A 90:10 polarization-maintaining fiber beam splitter (BS) combined with a power monitor (PWM) is used to apply feedback to the dc-bias port of the IM so as to maintain a constant $22 \mathrm{~dB}$ extinction ratio [54]. In order to successfully execute the quantum teleportation protocol, photons from Alice and Bob must be indistinguishable in all degrees of freedom (see Sec. III B). Hence, the optical pulses at the output of the IM are band-pass filtered using a 2-GHz-bandwidth (FWHM) fiber Bragg grating (FBG) centered at $1536.5 \mathrm{~nm}$ to match the spectrum of the photons from the entangled pair source (described in Sec. II B). Furthermore, the polarization of Alice's photons is determined by a manual polarization controller (POC) in conjunction with a polarizing beam splitter (PBS) at Charlie. Finally, the optical pulses from Alice are attenuated to the single-photon level by a variable optical attenuator (VOA), to approximate photonic time-bin qubits of the form $|A\rangle=\gamma|e\rangle_{A}+\sqrt{1-\gamma^{2}}|l\rangle_{A}$, where the late state $|l\rangle_{A}$ arrives 2 ns after the early state $|e\rangle_{A}$ and $\gamma$ is real and set to be either 1,0 , or $1 / \sqrt{2}$ to generate $|e\rangle_{A},|l\rangle_{A}$, or $|+\rangle_{A}=\left(|e\rangle_{A}+|l\rangle_{A}\right) / \sqrt{2}$, respectively, depending on the experiment. The complex relative phase is absorbed into the definition of $|l\rangle_{A}$. The duration of each time bin is 800 ps.

\section{B. Bob: entangled qubit generation and teleported-qubit measurement}

Similar to Alice, one (two) optical pulse(s) with a FWHM of approximately 65 ps is (and separated by $2 \mathrm{~ns}$ are) created using a $1536.5-\mathrm{nm} \mathrm{cw}$ laser in conjunction with a lithium niobate IM driven by an AWG, while the 90:10 BS and PWM are used to maintain an extinction ratio of at least $20 \mathrm{~dB}$. An erbium-doped fiber amplifier (EDFA) is used after the IM to boost the pulse power and thus maintain a high output rate of photon pairs.

The output of the EDFA is sent to a type- 0 periodically poled lithium niobate (PPLN) waveguide for secondharmonic generation (SHG), up-converting the pulses to $768.25 \mathrm{~nm}$. The residual light at $1536.5 \mathrm{~nm}$ is removed by a 768 -nm band-pass filter with an extinction ratio $\geq 80$ $\mathrm{dB}$. These pulses undergo spontaneous parametric downconversion (SPDC) using a type-II PPLN waveguide coupled to a polarization-maintaining fiber (PMF), approximately producing either a photon pair $\mid$ pair $\rangle_{B}=|e e\rangle_{B}$ or the time-bin entangled state $\left|\phi^{+}\right\rangle_{B}=\left(|e e\rangle_{B}+|l l\rangle_{B}\right) / \sqrt{2}$, if one or two pulses, respectively, are used to drive the IM.

The ordering of the states refers to so-called signal and idler modes of the pair of which the former has parallel, and the latter orthogonal, polarization with respect to the axis of the PMF. As before, the relative phase is absorbed into the definition of $|l l\rangle_{B}$. Each photon is separated into different fibers using a PBS and spectrally filtered with
FBGs akin to that at Alice. Note that the bandwidth of the FBG is chosen as a trade-off between spectral purity and the generation rate of Bob's photons [55].

The photon in the idler mode is sent to Charlie for teleportation or HOM measurements (see Sec. III B) or to a Mach-Zehnder interferometer (MZI) for characterizations of the entangled state (see Sec. III A), with its polarization determined using a POC. The photon in the signal mode is sent to a MZI by way of a POC (and an additional $11 \mathrm{~km}$ of single-mode fiber for some measurements) and is detected by superconducting nanowire single-photon detectors (SNSPDs) [56] after high-pass filtering (HPF) to reject any remaining 768.25-nm light. The MZI and detectors are used for projection measurements of the teleported state, characterization of the time-bin entangled state, or measuring HOM interference at Charlie. The time of arrival of the photons is recorded by the DAQ subsystem using a time-to-digital converter (TDC) referenced to the clock signal from the AWG.

All SNSPDs are installed in a compact-sorption fridge cryostat [57], which operates at a temperature of $0.8 \mathrm{~K}$ for typically $24 \mathrm{~h}$ before a required 2-h downtime. Our SNSPDs are developed at the Jet Propulsion Laboratory and have detection efficiencies between 76 and $85 \%$, with low dark-count rates of 2-3 Hz. The FWHM temporal resolution of all detectors is between 60 and $90 \mathrm{ps,} \mathrm{while} \mathrm{their}$ recovery time is approximately $50 \mathrm{~ns}$. A detailed description of the SNSPDs and the associated setup is provided in Appendix A 2.

The MZI has a path-length difference of 2 ns and is used to perform projection measurements of $|e\rangle_{B},|l\rangle_{B}$, and $\left(|e\rangle_{B}+e^{i \varphi}|l\rangle_{B}\right) / \sqrt{2}$, by detecting photons at three distinct arrival times in one of the outputs and varying the relative phase $\varphi$ [32]. Detection at the other output yields the same measurements except with a relative phase of $\varphi+\pi$. Using a custom temperature-feedback system, we slowly vary $\varphi$ for up to 15-h time intervals to collect all measurements, which is within the cryostat hold time. Further details of the MZI setup are described in Appendix A 3.

\section{Charlie: Bell-state measurement}

Charlie consists of a 50:50 polarization-maintaining fiber BS, with relevant photons from the Alice and Bob subsystems directed to each of its inputs via a PBSs and optical fiber. The photons are detected at each output with an SNSPD after HPFs, with their arrival times recorded using the DAQ, as was done at Bob. Teleportation is facilitated by measurement of the $\left|\Psi^{-}\right\rangle_{A B}=\left(|e l\rangle_{A B}-\right.$ $\left.|l e\rangle_{A B}\right) / \sqrt{2}$ Bell state, which corresponds to the detection of a photon in $|e\rangle$ at one detector followed by the detection of a photon in $|l\rangle$ at the other detector after Alice and Bob's (indistinguishable) qubits arrive at the BS [58]. Projection on the $\left|\Psi^{-}\right\rangle_{A B}$ state corresponds to teleportation of $|A\rangle$ up 
to a known local unitary transformation, i.e., our system produces $-i \sigma_{y}|A\rangle$, with $\sigma_{y}$ being the Pauli $y$ matrix.

\section{EXPERIMENTAL RESULTS}

Prior to performing quantum teleportation, we measure some key parameters of our system that underpin the teleportation fidelity. Specifically, we determine the fidelity of the entangled state produced by Bob by measuring the entanglement visibility $V_{\text {ent }}$ [59] and also determine to what extent Alice and Bob's photons are indistinguishable at Charlie's BS using the HOM effect [51].

\section{A. Entanglement visibility}

The state $\mid$ pair $\rangle_{B}$ (and hence the entangled state $\left|\phi^{+}\right\rangle_{B}$ ) described in Sec. II B is idealized. In reality, the state produced by Bob is better approximated by a two-mode squeezed vacuum state $|\mathrm{TMSV}\rangle_{B}=$ $\sqrt{1-p} \sum_{n=0}^{\infty} \sqrt{p}^{n}|n n\rangle_{B}$ after the FBG filter and neglecting loss [60]. Here, $n$ is the number of photons per temporal mode (or qubit) and $p$ is the emission probability of a single pair per mode (or qubit), with the state ordering referring to the signal and idler modes. However, $|\mathrm{TMSV}\rangle_{B}$ approximates a photon pair for $p \ll 1$, with a $p \approx \mu_{B}$ mean number of pairs per mode (or qubit), conditioned on measurement of a pair such that the $n=0$ term is eliminated. As a compromise between the pair-creation rate $\propto p$ and the quality of entanglement, here and henceforth we set the mean photon number of our pair source to be $\mu_{B}=$ $(8.0 \pm 0.4) \times 10^{-3}$ per time bin, which is feasible because of the exceptionally low dark counts of our SNSPDs. The measurement of $\mu_{B}$ is outlined in Appendix B.

We generate $\left|\phi^{+}\right\rangle_{B}$ and measure $V_{\text {ent }}$ by directing the idler photon to the second input port of the MZI, slightly modifying the setup of Fig. 1. The idler photon is delayed compared to the signal, allowing unambiguous measurement of each qubit. We vary $\varphi$ and project each qubit of the entangled state onto phase-varied superpositions of $|e\rangle$ and $|l\rangle$ by accumulating coincidence events of photons at both the outputs of the interferometer [59].

The results shown in Fig. 2 are fitted proportional to $1+V_{\text {ent }} \sin (\omega T+\Phi)$, where $V_{\text {ent }}=\left(R_{x}-R_{n}\right) /\left(R_{x}+R_{n}\right)$, with $R_{x(n)}$ denoting the maximum (minimum) rate of coincidence events [59], $\omega$ and $\Phi$ are unconstrained constants, and $T$ is the temperature of the MZI, finding $V_{\text {ent }}=96.4 \pm$ $0.3 \%$.

The deviation from unit visibility is mainly due to nonzero multiphoton emissions [61], which is supported by an analytical model that includes experimental imperfections [62]. Nonetheless, this visibility is far beyond the $1 / 3$ required for nonseparability of a Werner state [63] and the locality bound of $1 / \sqrt{2}[59,64]$. Furthermore, it predicts a fidelity $F_{\text {ent }}=\left(3 V_{\text {ent }}+1\right) / 4=97.3 \pm .2 \%$ with respect to $\left|\phi^{+}\right\rangle$[63] and hence is sufficient for quantum teleportation.

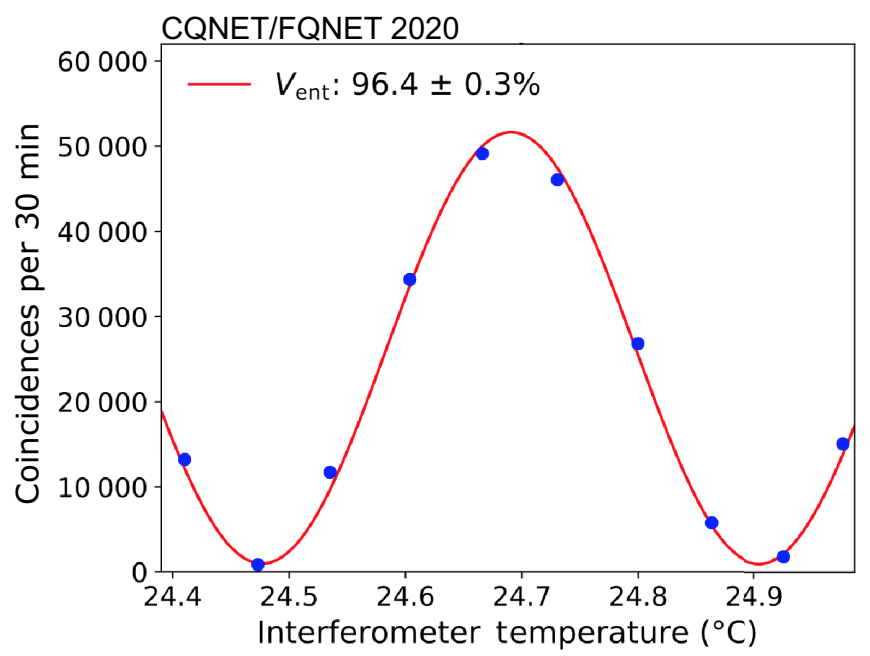

FIG. 2. Entanglement visibility. The temperature of the interferometer is varied to reveal the expected sinusoidal variations in the rate of coincidence events. A fit reveals the entanglement visibility $V_{\text {ent }}=96.4 \pm 0.3 \%$ (see the main text for details). The uncertainties here and in all measurements are calculated assuming Poisson statistics.

\section{B. HOM interference visibility}

The BSM relies on quantum interference of photons from Alice and Bob. This is ensured by the BS at Charlie, precise control of the arrival time of photons with IMs, identical FBG filters, and POCs (with PBSs) to provide the required indistinguishability. The degree of interference is quantified by way of the HOM interference visibility $V_{\text {HOM }}=\left(R_{d}-R_{i}\right) / R_{d}$, with $R_{d(i)}$ denoting the rate of coincident detections of photons after the BS when the photons are rendered as distinguishable (indistinguishable) as possible [51]. Completely indistinguishable single photons from Alice and Bob may yield $V_{\mathrm{HOM}}=1$. However, in our system, Alice's qubit is approximated from a coherent state $|\alpha\rangle_{A}=\mathrm{e}^{-|\alpha|^{2} / 2} \sum_{n=0}^{\infty}\left(\alpha^{n} / \sqrt{n !}\right)|n\rangle_{A}$ with $\alpha \ll 1$, akin to how Bob's pair is approximated from $|\mathrm{TMSV}\rangle_{B}$ (see Sec. III A), with $\mu_{A}=|\alpha|^{2}$ being Alice's mean photon number per mode (or qubit) [60]. Therefore, the contribution of undesired photons from Alice and Bob lowers the maximum achievable $V_{\mathrm{HOM}}$ below unity, with a further reduction if the interfering photons are not completely indistinguishable. The dependence of $V_{\mathrm{HOM}}$ with varied $\mu_{A}$ and $\mu_{B}$, including effects of losses or distinguishable photons in our system, is analytically modeled in Ref. [62] and briefly discussed in Sec. IV.

We measure $V_{\text {HOM }}$ by slightly modifying the setup of Fig. 1. We prepare $|A\rangle=|e\rangle_{A}$ with $\mu_{A}=2.6 \times 10^{-3}$ and Bob as $\mid$ pair $\rangle_{B}$ and direct Alice's photon and Bob's idler to Charlie, with Bob's signal bypassing the MZI to be directly measured by an SNSPD. Alice's IM is used to introduce distinguishability by way of a relative difference 
in arrival time $\Delta t_{A B}$ of Alice and Bob's photons at Charlie's BS. Using Charlie's SNSPDs and the third detector at Bob, a threefold coincidence detection rate is measured for varying $\Delta t_{A B}$, with results shown in Fig. 3(a). Since the temporal profiles of our photons are approximately Gaussian, we fit our results to $A\left[1-V_{\mathrm{HOM}} \exp \left(-\Delta t_{A B}^{2} / 2 \sigma^{2}\right)\right]$, where $A$ is the maximum coincidence rate when the photons are completely distinguishable and $\sigma=300 \mathrm{ps}$ is the $1 / e$ temporal duration of the optical pulses $[51,65]$, finding $V_{\mathrm{HOM}}=70.9 \pm 1.9 \%$. The maximum $V_{\mathrm{HOM}}$ for this experiment is $83.5 \%$ if the photons are completely indistinguishable [62], with the difference ascribed to slight distinguishability between our photons as supported by the further measurements and analytical modeling in Sec. IV. Improvements to our system to remove this distinguishability are discussed in Sec. V.

To test our system for quantum teleportation over long distances, we introduce the aforementioned 22-, 11-, and $11-\mathrm{km}$ lengths of single-mode fiber between Alice and Charlie, Bob and Charlie, and in the path of Bob's signal photon, respectively, repeat our measurement of $V_{\mathrm{HOM}}$, and fit the results as before [see Fig. 3(b)]. We find $V_{\mathrm{HOM}}=$ $63.4 \pm 5.9 \%$, which is consistent with the maximum $V_{\mathrm{HOM}}$ that we expect when including the impact of the additional 5.92-(2.56-)dB loss between Charlie and Alice (Bob) as well as the effect of photon distinguishability (analyzed in Sec. IV). This suggests that, importantly, the additional fiber does not introduce any further distinguishability (that we cannot account for), thereby supporting our system's use in quantum networking. Overall, the presence of clear HOM interference suggests that our system (with or without the additional fiber) introduces relatively little in the way of imperfections that can negatively impact the BSM and hence the fidelity of quantum teleportation.

\section{Quantum teleportation}

We now perform quantum teleportation of the time-bin qubit basis states $|e\rangle,|l\rangle$ and $|+\rangle$, so as to measure the teleportation fidelities, $F_{e}, F_{l}$, and $F_{+}$, respectively, of the teleported states with respect to their ideal counterparts, up to the local unitary introduced by the BSM (see Sec. II C). Since measurement of $|+\rangle$ in our setup by symmetry is equivalent to any state of the form $\left(|e\rangle+e^{i \varphi}|l\rangle\right) / \sqrt{2}$ (and, in particular, the remaining three basis states $(|e\rangle-$ $|l\rangle) / \sqrt{2}$ and $(|e\rangle \pm i|l\rangle) / \sqrt{2})$, we may determine the average teleportation fidelity $F_{\text {avg }}=\left(F_{e}+F_{l}+4 F_{+}\right) / 6$ of any time-bin qubit.

First, we prepare $|e\rangle_{A}$ and $|l\rangle_{A}$ with $\mu_{A}=3.53 \times 10^{-2}$, with Bob's idler bypassing the MZI to be detected by a single SNSPD. We measure $F_{e}=95 \pm 1 \%$ and $F_{l}=$ $96 \pm 1 \%$, conditioned on a successful measurement of $\left|\Psi^{-}\right\rangle_{A B}$ at Charlie, with the fidelity limited by multiphoton events in Alice and Bob's qubits and dark counts of the SNSPDs [62]. We then repeat the measurement with
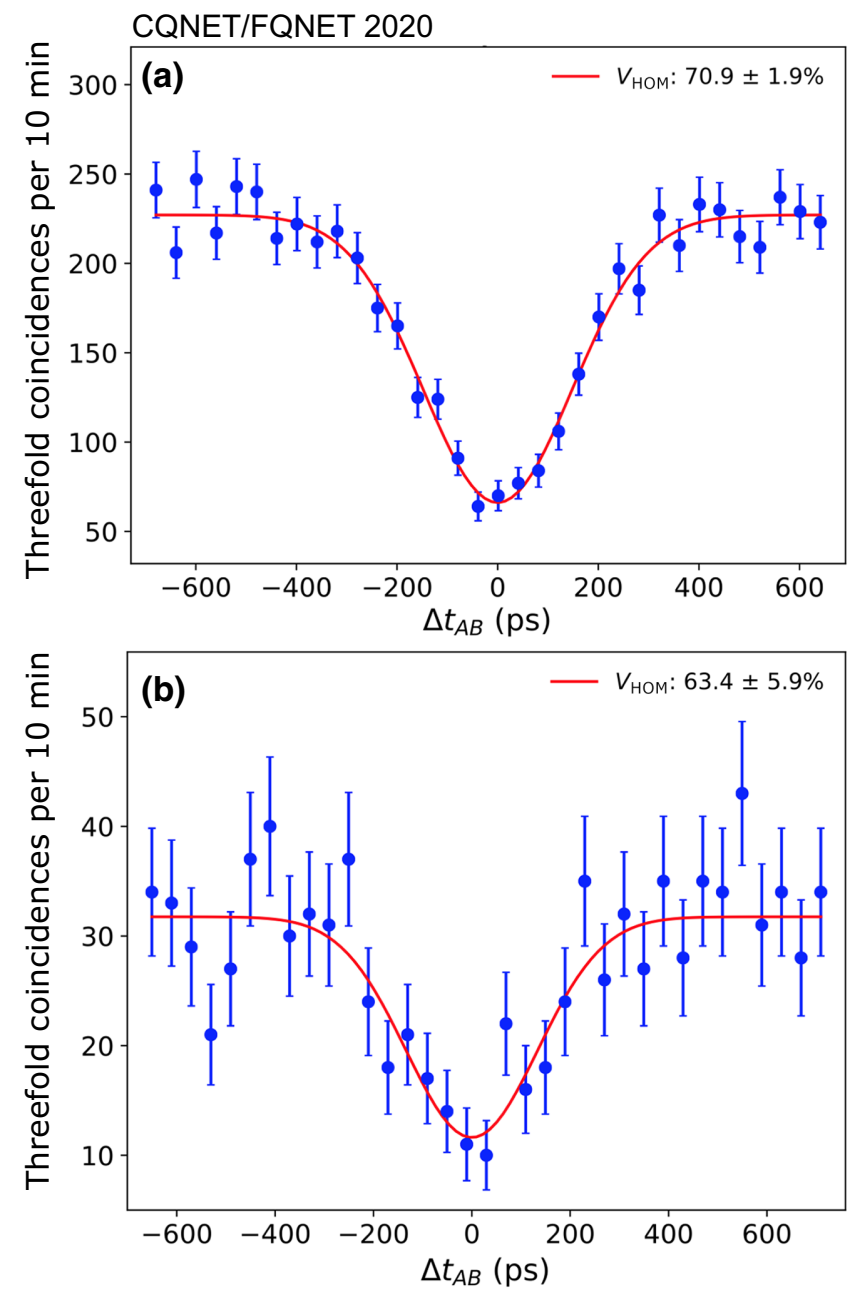

FIG. 3. Hong-Ou-Mandel (HOM) interference. A relative difference in arrival time is introduced between photons from Alice and Bob at Charlie's BS. HOM interference produces a reduction of the threefold coincidence detection rate of photons as measured with SNSPDs after Charlie's BS and at Bob. A fit reveals (a) $V_{\mathrm{HOM}}=70.9 \pm 1.9 \%$ and (b) $V_{\mathrm{HOM}}=63.4 \pm 5.9 \%$ when lengths of fiber are added (see the main text for details).

$\mu_{A}=9.5 \times 10^{-3}$ after inserting the aforementioned 44-km length of fiber as before to emulate Alice, Charlie, and parts of Bob being separated by long distances. This gives $F_{e}=98 \pm 1 \%$ and $F_{l}=98 \pm 2 \%$, with no reduction from the additional fiber loss owing to our low-noise SNSPDs.

Next, we prepare $|+\rangle_{A}$ with $\mu_{A}=9.38 \times 10^{-3}$, insert the MZI and, conditioned on the BSM, we measure $F_{+}=$ $\left(1+V_{+}\right) / 2=84.9 \pm 0.5 \%$ by varying $\varphi$. Here, $V_{+}=$ $69.7 \pm 0.9 \%$ is the average visibility obtained by fits to the resultant interference measured at each output of the MZI, as shown in Fig. 4(a). The reduction in fidelity from unity is due to multiphoton events and distinguishability, consistent with that inferred from HOM interference, as supported by further measurements and analytical modeling in Sec. IV. 

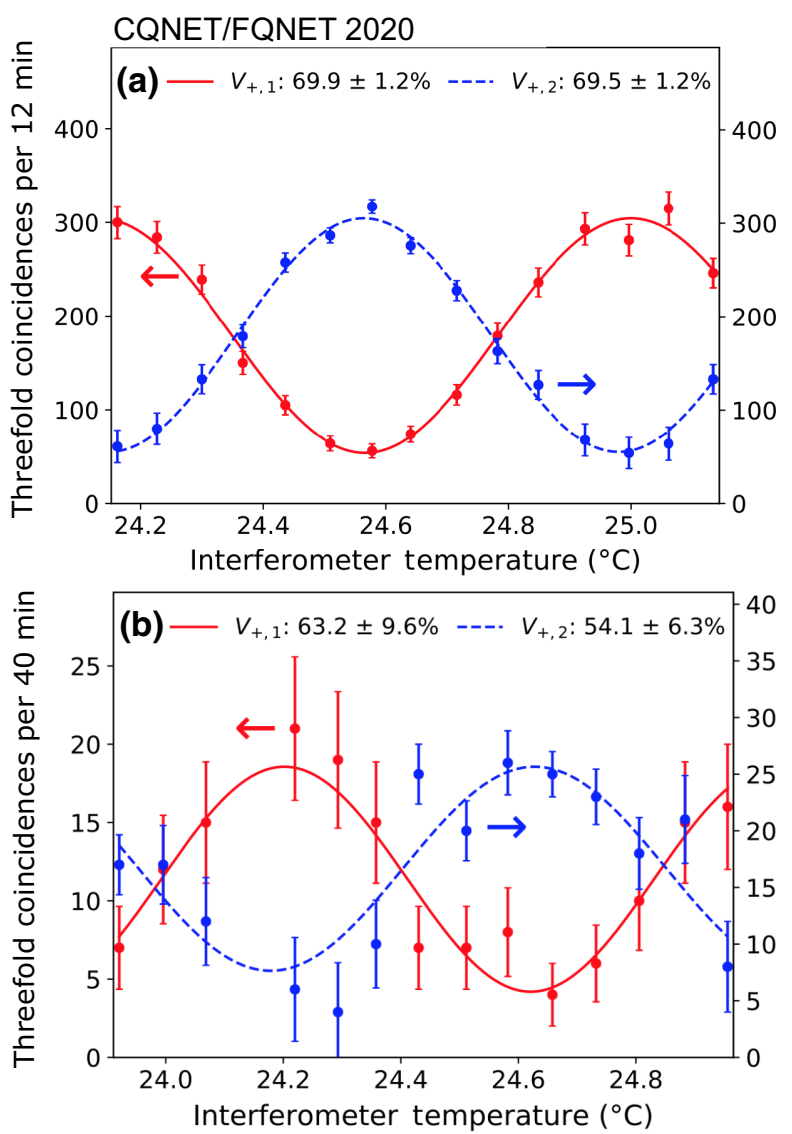

FIG. 4. The quantum teleportation of $|+\rangle$. Teleportation is performed (b) with and (a) without an additional $44 \mathrm{~km}$ of single-mode fiber inserted into the system. The temperature of the inteferometer is varied to yield a sinusoidal variation of the threefold coincidence rate at each output of the MZI (blue and red points). A fit of the visibilities (see Sec. III A) measured at each output $\left(V_{+, 1}, V_{+, 2}\right)$ of the MZI gives an average visibility $V_{+}=\left(V_{+, 1}+V_{+, 2}\right) / 2$ of (a) $69.7 \pm 0.91 \%$ without the additional fiber and (b) $58.6 \pm 5.7 \%$ with the additional fiber.

The measurement is repeated with the additional long fiber, giving $V_{+}=58.6 \pm 5.7 \%$ and $F_{+}=79.3 \pm 2.9 \%$, with the results and the corresponding fit shown in Fig. 4(b). The reduced fidelity is likely due to aforementioned polarization variations over the long fibers, consistent with the reduction in HOM interference visibility, and is exacerbated here owing to the less than ideal visibility of the MZI over long measurement times (see Appendix A 3).

The results yield $F_{\text {avg }}=89 \pm 1 \%(86 \pm 3 \%)$ without (with) the additional fiber, which is significantly above the classical bound of $2 / 3$, implying strong evidence of quantum teleportation [66] and limited from unity by multiphoton events, distinguishability, and polarization variations, as mentioned [62].

To glean more information about our teleportation system beyond the fidelity, we reconstruct the density matrices of the teleported states using a maximum-likelihood QST
[52] described in Appendix C. The results of the QST with and without the additional fiber lengths are summarized in Figs. 8 and 9, respectively. As can be seen, the diagonal elements for $|+\rangle$ are very close to the expected value, indicating the preservation of probabilities for the basis states of $|e\rangle$ and $|l\rangle$ after teleportation, while the deviation of the off-diagonal elements indicates the deterioration of coherence between the basis states. The decoherence is attributed to multiphoton emissions from our entangled pair source and distinguishability, consistent with the aforementioned teleportation fidelities of $|+\rangle_{A}$, and is further discussed in Sec. IV. Finally, we do also extract the teleportation fidelity from these density matrices, finding the results shown in Fig. 5 and $F_{\text {avg }}=89 \pm 1 \%(88 \pm 3 \%)$

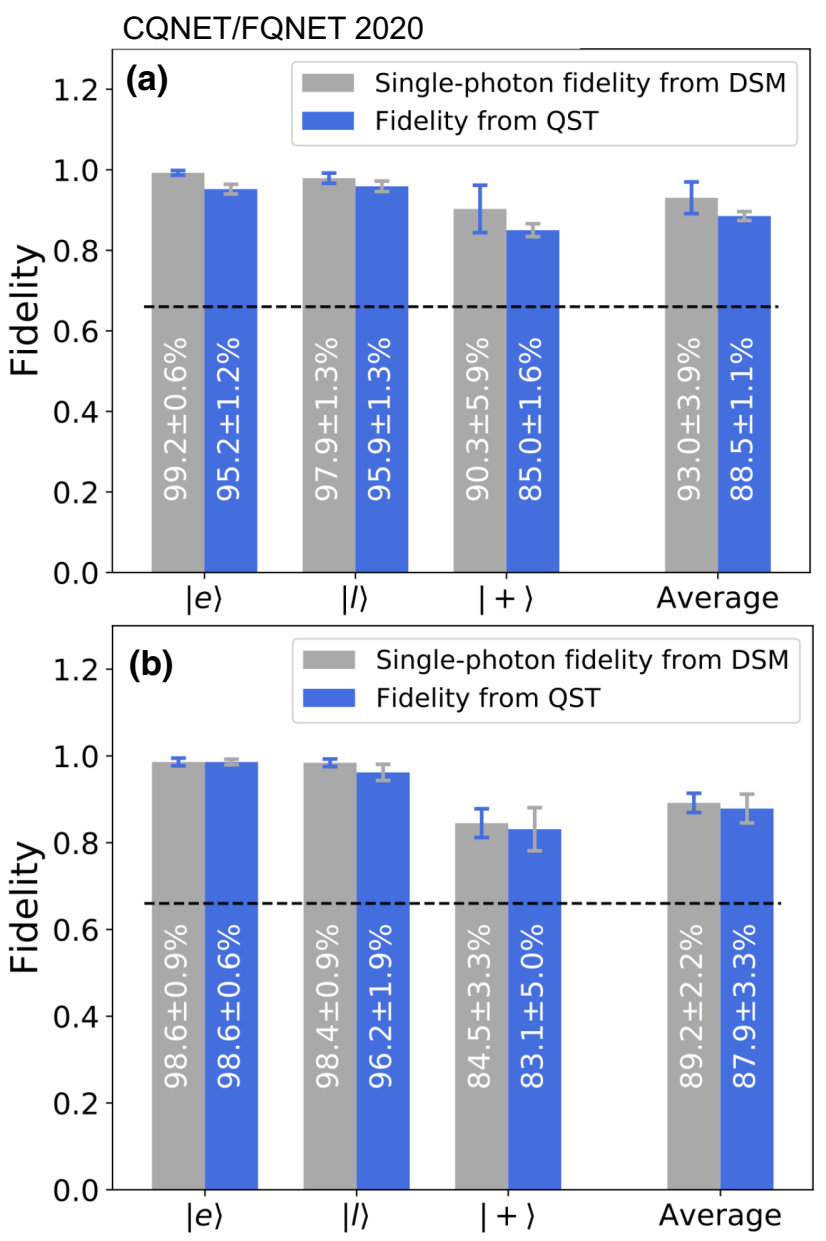

FIG. 5. Quantum teleportation fidelities for $|e\rangle_{A},|l\rangle_{A}$, and $|+\rangle_{A}$, including the average fidelity. The dashed line represents the classical bound. Fidelities using QST are shown using blue bars, while the minimum fidelities for qubits prepared using $|n=1\rangle, F_{e}^{d}, F_{l}^{d}$, and $F_{+}^{d}$, including the associated average fidelity $F_{\text {avg }}^{d}$, respectively, using a DSM are shown in gray. (a),(b) The results without and with additional fiber, respectively. The uncertainties are calculated using Monte Carlo simulations with Poissonian statistics. 
without (with) the fiber spools, which are consistent with previous measurements given the similar $\mu_{A}$ used for QST.

The use of weak coherent states instead of ideal single photons reduces the teleportation fidelity because of multiphoton events. This effect is further discussed in Sec. IV. We also point out that the $2 / 3$ classical bound may only be applied if Alice prepares her qubits using genuine single photons, i.e., $|n=1\rangle$, rather than using $|\alpha \ll 1\rangle$ as we do in this work [67]. As a way to account for the photon statistics of Alice's qubits, we turn to an analysis using decoy states.

\section{Teleportation fidelity using decoy states}

To determine the minimum teleportation fidelity of qubits prepared using single photons, we use a DSM [53] and follow the approach of Refs. [33,68]. Decoy states, which are traditionally used in quantum key distribution to defend against photon-number splitting attacks, are qubits encoded into coherent states $|\alpha\rangle$ with varying mean photon number $\mu_{A}=|\alpha|^{2}$. By measuring fidelities of the teleported qubits for different $\mu_{A}$, the DSM allows us to calculate a lower bound $F_{A}^{d}$ on the teleportation fidelity if Alice had encoded her qubits using $|n=1\rangle$.

We prepare decoy states $|e\rangle_{A},|l\rangle_{A}$, and $|+\rangle_{A}$ with varying $\mu_{A}$, as listed in Table I, and perform quantum teleportation both with and without the added fiber, with teleportation fidelities shown in Table I. From these results, we calculate $F_{A}^{d}$ as shown in Fig. 5, with $F_{\text {avg }}^{d} \geq 93 \pm 4 \%$ ( $F_{\mathrm{avg}}^{d} \geq 89 \pm 2 \%$ ) without (with) the added fiber, which significantly violates the classical bound and the bound of 5/6 given by an optimal symmetric universal cloner $[69,70]$, clearly demonstrating the capability of our system for high-fidelity teleportation. As depicted in Fig. 5, these fidelities nearly match the results we obtain without decoy

TABLE I. The teleportation fidelities with (right column) and without (center column) the 44-km length of fiber for Alice's qubit states prepared with varying $\mu_{A}$. The mean photon numbers and fidelities for vacuum states with fiber are assumed to be zero and $50 \%$, respectively.

\begin{tabular}{lccccc}
\hline \hline \multirow{2}{*}{ Qubit } & \multicolumn{2}{c}{ Without long fiber } & & \multicolumn{2}{c}{ With long fiber } \\
\cline { 2 - 3 } \cline { 5 - 6 } & $\mu_{A}\left(\times 10^{-3}\right)$ & $F_{A}^{d}(\%)$ & & $\mu_{A}\left(\times 10^{-3}\right)$ & $F_{A}^{d}(\%)$ \\
\hline$|e\rangle_{A}$ & 3.53 & $95.2 \pm 1$ & & 26.6 & $95.7 \pm 1.5$ \\
& 1.24 & $86.7 \pm 2$ & & 9.01 & $98.4 \pm 1.1$ \\
& 0 & $52.8 \pm 3.4$ & & $\ldots$ & $\ldots$ \\
$|l\rangle_{A}$ & 3.53 & $95.9 \pm 1$ & & 32.9 & $98.6 \pm 0.7$ \\
& 1.24 & $90.5 \pm 2$ & & 9.49 & $98.4 \pm 1.6$ \\
$|+\rangle_{A}$ & 0 & $52.8 \pm 3.4$ & & $\ldots$ & $\ldots$ \\
& 9.38 & $84.7 \pm 1.1$ & & 29.7 & $73.6 \pm 3.0$ \\
& 2.01 & $83.2 \pm 3.6$ & & 10.6 & $82.21 \pm 3.9$ \\
\hline \hline
\end{tabular}

states within statistical uncertainty. This is due to the suitable $\mu_{A}$, as well as low $\mu_{B}$ and SNSPD dark counts in our previous measurements [62].

\section{ANALYTICAL MODEL AND SIMULATION}

As our measurements have suggested, multiphoton components in, and distinguishability between, Alice and Bob's qubits reduce the values of key metrics including HOM interference visibility and, consequently, quantum teleportation fidelity. To capture these effects in our model, we employ a Gaussian-state characteristic-function method developed in Ref. [62], which is enabled by work in Ref. [71]. This approach is well suited to analyze our system because the quantum states, operations, and imperfections (including losses, dark counts, etc.) of the experiment can be fully described using Gaussian operators (see, e.g., Ref. [72]). We now briefly outline the model of Ref. [62] and employ it to estimate the amount of indistinguishability $\zeta$ between Alice and Bob's qubits in our measurements of HOM interference and quantum teleportation.

The distinguishability in any degree of freedom may be modeled by introducing a virtual BS of transmittance $\zeta$ into the paths of Alice and Bob's relevant photons. As shown in Fig. 6, the indistinguishable components of incoming photon modes are directed toward Charlie's BS, where they interfere, whereas distinguishable components are mixed with vacuum at the BS and do not contribute to interference. Here, $\zeta=1(\zeta=0)$ corresponds to the case when both incoming photons are perfectly indistinguishable (distinguishable). Now we may calculate the probability of a threefold coincidence detection event $P_{3 f}$ between $D_{1}, D_{2}$ (Charlies' detectors) and $D_{3}$ (detects Bob's signal photon) for a given qubit state $\rho_{A B}$ from Alice and Bob:

$$
\begin{aligned}
P_{3 f}= & \operatorname{Tr}\left\{\rho_{A B}\left(\mathbb{I}-(|0\rangle\langle 0|)_{\hat{a}_{1}, \hat{a}_{2}, \hat{a}_{3}}^{\otimes^{3}}\right)\right. \\
& \left.\otimes\left(\mathbb{I}-(|0\rangle\langle 0|)_{\hat{b}_{1}, \hat{b}_{2}, \hat{b}_{3}}^{\otimes^{3}}\right) \otimes\left(\mathbb{I}-(|0\rangle\langle 0|)_{\hat{c}}\right)\right\},
\end{aligned}
$$

where the $\hat{a}$ and $\hat{b}$ operators refer to modes, which originate from Alice and Bob's virtual BSs and are directed to $D_{1}$ and $D_{2}$, respectively, and $\hat{c}$ corresponds to Bob's idler mode, which is directed to $D_{3}$ (see Fig. 6). This allows the derivation of an expression for the HOM interference visibility

$$
V_{\mathrm{HOM}}(\zeta)=\left[P_{3 f}(0)-P_{3 f}(\zeta)\right] / P_{3 f}(0),
$$

consistent with that introduced in Sec. III B. Since Alice and Bob ideally produce $\rho_{A B}=(|\alpha\rangle\langle\alpha|) \otimes\left(\left|\Psi_{\mathrm{TMSV}}\right\rangle\right.$ $\left.\left\langle\Psi_{\mathrm{TMSV}}\right|\right)$ and recognizing that all operators in $P_{3 f}$ are 


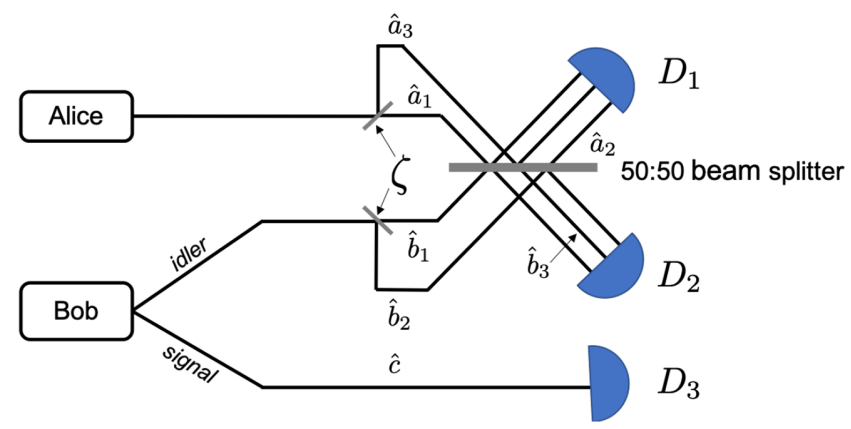

FIG. 6. A schematic depiction of the distingushability between Alice and Bob's photons at Charlie's BS. The distinguishability is modeled by means of a virtual BS with a transmittance $\zeta$. Indistinguishable photons contribute to interference at the Charlie's BS while distinguishable photons are mixed with vacuum, leading to a reduction of HOM visibility and teleportation fidelity (see the main text for further details).

Gaussian, we analytically derive

$$
\begin{aligned}
P_{3 f}(\zeta)= & 1-2 \frac{\exp \left(-\frac{\left(\mu_{A} / 2\right)\left[1+\left(1-\zeta^{2}\right) \eta_{i} \mu_{B} / 2\right]}{1+\eta_{i} \mu_{B} / 2}\right)}{1+\eta_{i} \mu_{B} / 2} \\
& -\frac{1}{1+\eta_{s} \mu_{B}}+\frac{\exp \left(-\mu_{A}\right)}{1+\eta_{i} \mu_{B}} \\
& -\frac{\exp \left(-\mu_{A}\right)}{1+\left(1-\eta_{s}\right) \eta_{i} \mu_{B}+\eta_{s} \mu_{B}} \\
& +2 \frac{\exp \left(-\frac{\left(\mu_{A} / 2\right)\left[1+\left(1-\zeta^{2}\right)\left(1-\eta_{s}\right) \eta_{i} \mu_{B} / 2+\eta_{s} \mu_{B}\right]}{1+\left(1-\eta_{s}\right) \eta_{i} \mu_{B} / 2+\eta_{s} \mu_{B}}\right)}{1+\left(1-\eta_{s}\right) \eta_{i} \mu_{B} / 2+\eta_{s} \mu_{B}}
\end{aligned}
$$

for varied $\zeta$, where $\eta_{i}$ and $\eta_{s}$ are the transmission efficiencies of the signal and idler photons, including the detector efficiencies. We similarly calculate the impact of distinguishability on the teleportation fidelity of $|+\rangle$ :

$$
F(\zeta)=P_{3 f}\left(\zeta, \varphi_{\max }\right) /\left[P_{3 f}\left(\zeta, \varphi_{\max }\right)+P_{3 f}\left(\zeta, \varphi_{\min }\right)\right]
$$

where $\varphi_{\max }\left(\varphi_{\min }\right)$ is the phase of the MZI added into the path of the signal photon, corresponding to maximum (minimum) threefold detection rates.

To compare the model to our measurements, we use the experimental mean photon numbers for the photon-pair source $\eta_{i}=1.2 \times 10^{-2}$ and $\eta_{s}=4.5 \times 10^{-3}$, as determined by the method described in Appendix B. We then measure the teleportation fidelity of $|+\rangle$ and HOM interference visibility (keeping the MZI in the system to ensure that $\eta_{s}$ remains unchanged) for different values $\mu_{A}$. The results are plotted in Fig. 7. The data are then fitted to the expressions $V_{\mathrm{HOM}}(\zeta)$ and $F(\zeta)$ derived in our model and graphed in Fig. 7. The maximum teleportation fidelity and

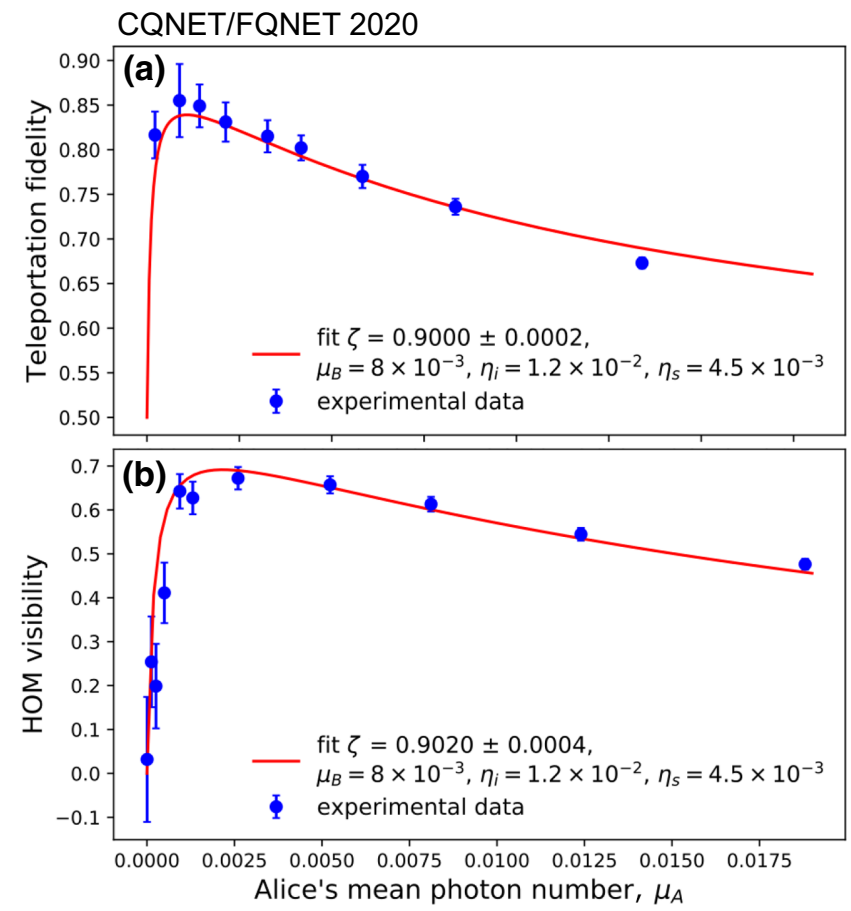

FIG. 7. The evaluation of the photon indistinguishability using an analytical model. (a) The quantum teleportation fidelity of $|+\rangle$. (b) The HOM interference visibility, each with varied mean photon number $\mu_{A}$ of Alice's qubits. Fits of analytical models to the data reveal $\zeta=90 \%$ indistinguishability between Alice and Bob's photons at Charlie's BS. Bob produces $\mu_{B}$ photon pairs on average, while $\eta_{i}$ and $\eta_{s}$ are the probabilities for an individual idler (signal) photon to arrive at Charlie's BS and be detected at Bob's detector, respectively.

HOM visibility are approximately 0.85 and approximately 0.65 , respectively, and are maximized around $\mu_{A}=0.001$. The teleportation fidelity and HOM visibility decrease to 0.5 and 0.0 , respectively, when $\mu_{A}=0$ corresponding to a vacuum input from Alice. The decrease at large values of $\mu_{A}$ is predominantly due to the multiphoton events from Alice.

The fitted curves are in very good agreement with our experimental values and consistently yield a value of $\zeta=90 \%$ for both measurement types. This implies that we have only a small amount of residual distinguishability between Alice and Bob's photons. The potential effects leading to this distinguishability are discussed in Sec. V.

Overall, our analytical model is consistent with our experimental data [62] and our experimental setup allows high-fidelity teleportation of arbitrary time-bin qubits in the regime of $\mu_{A} \ll 1$, which is the parameter space most often used in quantum networking protocols (e.g., key distribution). Our model thus offers a practical way to determine any underlying distinguishability in a deployed 
network where a full characterization of the properties of Alice and Bob's photons may not be possible.

\section{DISCUSSION AND OUTLOOK}

We demonstrate quantum teleportation systems for photonic time-bin qubits at a quantum channel- and devicecompatible wavelength of $1536.5 \mathrm{~nm}$ using a fiber-based setup comprising state-of-the-art SNSPDs and off-the-shelf components. We measure an average fidelity above $90 \%$ using QST and a decoy-state analysis with up to $44 \mathrm{~km}$ of single-mode fiber in our setup. Our results are further supported by an analytical model in conjunction with measurements of the entanglement and HOM interference visibilities.

The decoy-state analysis indicates that the maximum teleportation fidelity is currently restricted by that of the teleported qubits prepared in superposition states, for which a $10 \%$ distinguishability between the qubits undergoing BSM and the contribution of multiple photon pairs play the largest role. Our model predicts that the average fidelity will increase to $95 \%$ with completely indistinguishable photons, while fidelities close to unity can be achieved with a lowered mean number of photon pairs. Alternatively, we may replace our SNSPDs with efficient photon-number-resolving (PNR) SNSPDs [73] to allow postselection of multiphoton events at the MZI or BSM [74]. Both approaches must be accompanied by increased coupling efficiencies of the pair source beyond the current approximately $1 \%$, either to maintain realistic teleportation rates (above the system noise and current rate of phase drift of the MZI) or to derive any advantage from PNR capability.

As suggested by the width of our HOM interference fringe - which predicts an average photon bandwidth of $0.44 / \sigma \sim 1.5 \mathrm{GHz}$ (see Sec. III B), i.e., less than the $2 \mathrm{GHz}$ bandwidth of our FBGs - the indistinguishability in our system could be limited by the large difference in the bandwidth between the photons originating from the SPDC $(>100 \mathrm{GHz})$ and those generated at Alice by the IM (15 GHz), leading to nonidentical filtering by the FBG. This can be improved by narrower FBGs or by using a more broadband pump at Alice [e.g., using a mode-locked laser or a higher-bandwidth IM (say, > $50 \mathrm{GHz}$ ), which is commercially available]. Alternatively, pure photon pairs may be generated by engineered phase matching (see, e.g., Ref. [75]). Distinguishability owing to nonlinear modulation during the SHG process could also play a role [76]. The origin of distinguishability in our system, whether due to imperfect filtering or other device imperfections (e.g., PBS or BS) will be studied in future work. The coupling loss can be minimized to less than a few decibels overall by improved fiber-to-chip coupling, lower-loss components of the FBGs (e.g., the required isolator), spliced fiber connections, and reduced losses within our MZI. Note that our current coupling efficiency is equivalent to approximately $50 \mathrm{~km}$ of single-mode fiber, suggesting that our system is well suited for quantum networks provided that the loss is reduced.

While the fidelities that we demonstrate are sufficient for several applications, the current teleportation rates with the $44 \mathrm{~km}$ length of fiber, which are approximately in the Hertz range, are still low. Higher repetition rates (e.g., through the use of high-bandwidth modulators with wide-band wavelength-division-multiplexed filters and low-jitter SNSPDs [77]), improvements to coupling and detector efficiencies, enhanced BSM efficiency with fast-recovery SNSPDs [78], or multiplexing in frequency [68] will all yield substantial increases in the teleportation rate. Note that increased repetition rates permit a reduction in the time-bin separation, which will allow us to construct the MZI on chip, providing exceptional phase stability and, hence, achievable fidelity. Importantly, the aforementioned increases in the repetition rate and efficiency are afforded by improvements in SNSPD technology that are currently being pursued with our Jet Propulsion Laboratory (JPL), NIST, and other academic partners.

Upcoming system-level improvements that we plan to investigate and implement include further automation by the implementation of free-running temporal and polarization feedback schemes to render the photons indistinguishable at the BSM [33,34]. Furthermore, several electrical components can be miniaturized, scaled, and made more cost effective (e.g., field-programmable gate arrays can replace the AWG). We note that our setup prototype will be easily extended to independent lasers at different locations, also with appropriate feedback mechanisms for spectral overlap $[79,80]$. These planned improvements are compatible with the data acquisition and control systems that were built for the systems and experiments at FQNET and CQNET presented in this work.

Overall, our high-fidelity teleportation systemsachieving state-of-the-art teleportation fidelities of timebin qubits - serve as a blueprint for the construction of quantum network test beds and eventually global quantum networks toward the quantum Internet. In this work, we present a complete analytical model of the teleporation system that includes imperfections and compare it with our measurements. Our implementation, using approaches from HEP experimental systems and real-world quantum networking, features near fully automated data acquisition, monitoring, and real-time data analysis. In this regard, our Fermilab and Caltech Quantum Networks serve as R\&D laboratories and prototypes toward real-world quantum networks. The high fidelities achieved in our experiments using practical and replicable devices are essential when expanding a quantum network to many nodes and enable the realization of more advanced protocols (see, e.g., Refs. $[18,81,82])$. 


\section{ACKNOWLEDGMENTS}

R.V., N.L., L.N., C.P., N.S., M.S., and S.X. acknowledge partial and S.D. full support from the AQT IN-QNET research program. R.V., N.L., L.N., C.P., N.S., M.S., S.X., and A.M. acknowledge partial support from the U.S. Department of Energy, Office of Science, High Energy Physics, QuantISED program grant, under Award No. DESC0019219 and Basic Energy Sciences, under Award No. DE-SC0020376 (HEADS-QON on transduction design and memory compatibility). A.M. is supported in part by the JPL President and Director's Research and Development Fund (PDRDF). C.P. further acknowledges partial support from Fermilab's Lederman Fellowship and Lab Directed R\&D (LDRD). D.O. and N.S. acknowledge partial support from the Natural Sciences and Research Council of Canada (NSERC). D.O. further acknowledges the Canadian Foundation for Innovation, Alberta Innovates, and Alberta Economic Development, Trade and Tourism's Major Innovation Fund. J.A. acknowledges support by a NASA Space Technology Research Fellowship. Part of the research was carried out at the Jet Propulsion Laboratory, California Institute of Technology, under a contract with the National Aeronautics and Space Administration (80NM0018D0004). We thank Jason Trevor (Caltech Lauritsen Laboratory for High Energy Physics), Nigel Lockyer and Joseph Lykken (Fermilab), Vikas Anant (PhotonSpot), Aaron Miller (Quantum Opus), Inder Monga and his ESnet group at Lawrence Berkeley National Laboratory (LBNL), the groups of Wolfgang Tittel and Christoph Simon at the University of Calgary, the groups of Nick Hutzler, Oskar Painter, Andrei Faraon, Manuel Enders, and Alireza Marandi at Caltech, Marko Loncar's group at Harvard, Artur Apresyan and the HL-LHC USCMS-MTD Fermilab group; Marco Colangelo (MIT); Tian Zhong (Chicago); and AT\&T's Soren Telfer, Rishi Pravahan, Igal Elbaz, Andre Feutch, and John Donovan. We acknowledge the enthusiastic support of the Kavli Foundation on funding quantum information science and technology (QIS\&T) workshops and events and the Brinson Foundation support, especially for students working at FQNET and CQNET. M.S. is especially grateful to Norm Augustine (Lockheed Martin), Carl Williams (NIST), and Joe Broz (SRI, QED-C); Hartmut Neven (Google Venice); Amir Yacoby and Misha Lukin (Harvard); Ned Allen (Lockheed Martin); Larry James and Ed Chow (JPL); the Quantum Communication Channels for Fundamental Physics (QCCFP) wormhole-teleportation team, especially Daniel Jafferis (Harvard) and Alex Zlokapa (Caltech), Mark Kasevich (Stanford), Ronald Walsworth (Maryland), Jun Yeh and Sae Woo Nam (NIST); Irfan Siddiqi (Berkeley); Prem Kumar (Northwestern), Saikat Guha (Arizona), Paul Kwiat (UIUC), Mark Saffman (Wisconsin), Jelena Vuckovic (Stanford) Jack Hidary (former googleX), and the quantum networking teams at Oak
Ridge National Laboratory (ORNL), Argonne National Laboratory (ANL), and Brookhaven National Laboratory (BNL), for productive discussions and interactions on quantum networks and communications.

\section{APPENDIX A: DETAILED DESCRIPTION OF EXPERIMENTAL COMPONENTS}

\section{Control systems and data acquisition}

Our system is built with a vision toward future replicability, with a particular emphasis on systems integration. Each of the Alice, Bob, and Charlie subsystems is equipped with monitoring and active-feedback stabilization systems (e.g., for the IM extinction ratio) or has a capability for remote control of the critical network parameters (e.g., varying the qubit generation time). Each subsystem has a central classical processing unit with the following functions: oversight of automated functions and workflows within the subsystem, data acquisition and management, and handling of input and output synchronization streams. As the quantum information is encoded in the time domain, the correct operation of the classical processing unit depends critically on the recorded time of arrival of the photons at the SNSPDs. Thus a significant effort is dedicated to building a robust DAQ subsystem capable of recording and processing the large volumes of timetagged signals generated by the SNSPDs and recorded by our TDCs at a high rate. The DAQ is designed to enable both real-time data analysis for prompt data-quality monitoring as well as postprocessing data analysis that allows us to achieve the best understanding of the data.

The DAQ system is built on top of the stand-alone Linux library of our commercial TDC. It records time tags whenever a signal is detected in any channel in coincidence with the reference $90-\mathrm{MHz}$ clock. The time tags are streamed to a personal computer, where they are processed in real-time and stored to disk for future analysis. A graphical user interface is developed, capable of real-time visualization and monitoring of photons detected while executing teleportation. It also allows for easy control of the time intervals used for each channel and configuration of the relevant coincidences between the different photon detection events across all TDC channels. We expect our DAQ subsystem to serve as the foundation for future real-world time-bin quantum networking experiments (see Sec. V).

\section{Superconducting nanowire single-photon detectors}

We employ amorphous tungsten silicide SNSPDs manufactured in the JPL Microdevices Laboratory for all measurements at the single-photon level (see Sec. II B) [56]. The entire detection system is customized for optimum autonomous operation in a quantum network. The SNSPDs are operated at $0.8 \mathrm{~K}$ in a closed-cycle sorption fridge [57]. 
The detectors have nanowire widths between 140 and 160 $\mathrm{nm}$ and are biased at a current of 8-9 $\mu \mathrm{A}$. The FWHM timing jitter (i.e., temporal resolution) for all detectors is between 60 and 90 ps (measured using a Becker \& Hickl SPC-150NXX time-tagging module). The system detection efficiencies (as measured from the fiber bulkhead of the cryostat) are between 76 and $85 \%$. The SNSPDs feature low dark-count rates between 2 and $3 \mathrm{~Hz}$, achieved by short-pass filtering of background black-body radiation through coiling of optical fiber to a $3-\mathrm{cm}$ diameter within the 40-K cryogenic environment, and an additional band-pass filter coating deposited on the detector fiber pigtails (by Andover Corporation). Biasing of the SNSPDs is facilitated by cryogenic bias tees with inductive shunts to prevent latching, thus enabling uninterrupted operation. The detection signals are amplified using Mini-Circuits ZX60-P103LN+ and ZFL-1000LN+ amplifiers at room temperature, achieving a total noise figure of $0.61 \mathrm{~dB}$ and a gain of $39 \mathrm{~dB}$ at $1 \mathrm{GHz}$, which enables the low system jitter. Note that FWHM jitter as low as $45 \mathrm{ps}$ is achievable with the system, by biasing the detectors at approximately $10 \mu \mathrm{A}$, at the cost of an elevated DCR on the order of 30 cps. Using commercially available components, the system is readily scalable to as many as 64 channels per cryostat, ideal for star-type quantum networks, with uninterrupted $24 / 7$ operation. The bulkiest component of the current system is an external helium compressor; however, compact rack-mountable versions are readily available [57].

\section{Interferometer and phase stabilization}

We use a commercial Kylia 04906-MINT MZI, which is constructed of free-space devices (e.g., mirrors and BSs) with a small form factor that fits into a handheld box. Light is coupled into and out of the MZI using polarizationmaintaining fiber, with a loss of approximately $2.5 \mathrm{~dB}$. The interferometer features an average visibility of $98.5 \%$ that is determined by directing $|+\rangle$ with $\mu_{A}=0.07$ into one of the input ports, measuring the fringe visibility on each of the outputs using an SNSPD. The relative phase $\varphi$ is controlled by a voltage-driven heater that introduces a small change in refractive index in one arm of the MZI. However, this built-in heater does not permit phase stability that is sufficient to measure high-fidelity teleportation, with the relative phase following the slowly varying ambient temperature of the room. To mitigate this instability, we build another casing, thermally isolating the MZI enclosure from the laboratory environment, and control the temperature via a closed-loop feedback control system based on a commercial thermoelectric cooler and a LTC1923 PID-controller. The temperature feedback is provided by a $10-\mathrm{k} \Omega$ NTC thermistor while the set point is applied with a programmable power supply. This control system permits us to measure visibilities by slowly varying $\varphi$ over time scales of up to $15 \mathrm{~h}$. We remark that no
TABLE II. Bob's photon-pair source is characterized by the measured mean photon number per time bin, $\mu_{B}$, and the rate of accidental and true-coincidence detections with a varied EDFA current.

\begin{tabular}{lccc}
\hline \hline $\begin{array}{l}\text { EDFA current } \\
(\mathrm{mA})\end{array}$ & $\begin{array}{c}\text { Coincidences } \\
(\text { per 10 s })\end{array}$ & $\begin{array}{c}\text { Accidentals } \\
(\text { per 10 s })\end{array}$ & $\begin{array}{c}\mu_{B} \\
\left(\times 10^{-3}\right)\end{array}$ \\
\hline 400 & $469.2 \pm 3.6$ & $1.8 \pm 0.3$ & $3.9 \pm 0.7$ \\
450 & $1156.3 \pm 4.9$ & $6.1 \pm 0.5$ & $5.3 \pm 0.4$ \\
500 & $1653.9 \pm 5.9$ & $9.5 \pm 0.6$ & $5.8 \pm 0.4$ \\
550 & $2095.8 \pm 6.6$ & $13.7 \pm 0.8$ & $6.5 \pm 0.4$ \\
575 & $2343.2 \pm 7.0$ & $17.7 \pm 0.9$ & $7.5 \pm 0.4$ \\
600 & $2548.7 \pm 7.3$ & $18.5 \pm 0.9$ & $8.0 \pm 0.4$ \\
\hline \hline
\end{tabular}

additional phase-control methods are used beyond that of temperature.

\section{APPENDIX B: ESTIMATION OF MEAN NUMBER OF PHOTON PAIRS AND TRANSMISSION EFFICIENCIES OF SIGNAL AND IDLER PHOTONS}

Using a method described in Ref. [59], we measure the mean number of photon pairs produced by Bob, $\mu_{B}$, as a function of the laser-excitation power before the PPLN waveguide used for SHG. To this end, we modify the setup of Fig. 1 and direct each of Bob's signal and idler photons to a SNSPD. We then measure detection events while varying the amplification of our EDFA by way of an applied current. We extract events when photon pairs that originate from the same clock cycle are measured in coincidence and when one photon originating from a cycle is measured in coincidence with a photon originating from a preceding or following clock cycle; in other words, we measure the so-called coincidence and accidental rates. The ratio of accidentals to coincidences approximates $\mu_{B} \ll 1$, with all results shown in Table II. For all measurements, we use $\mu_{B}=(8.0 \pm 0.4) \times 10^{-3}$ per time bin, which corresponds to an EDFA current of $600 \mathrm{~mA}$.

The transmission efficiencies of the signal and idler photons, $\eta_{s}$ and $\eta_{i}$, respectively, mentioned in Sec. IV, are determined by calculating the ratio of the independent rates of detection of the idler and signal photons, respectively, with the coincidence rate of the photons pairs (in the same clock cycle) [59]. We repeat the measurements using the setup shown in Fig. 1, which is that used to generate the results of Fig. 7 (i.e., we direct the signal and idler photons through the setup as if we are to perform teleportation). We find $\eta_{s}=4.5 \times 10^{-3}$ and $\eta_{i}=1.2 \times 10^{-2}$, which take into account losses between when the photons are produced and when they are detected by their respective SNSPDs.

\section{APPENDIX C: QUANTUM STATE TOMOGRAPHY}

We perform projection measurements on the teleported states $|e\rangle_{A},|l\rangle_{B}$, and $|+\rangle_{A}$ in all three of the qubit bases 

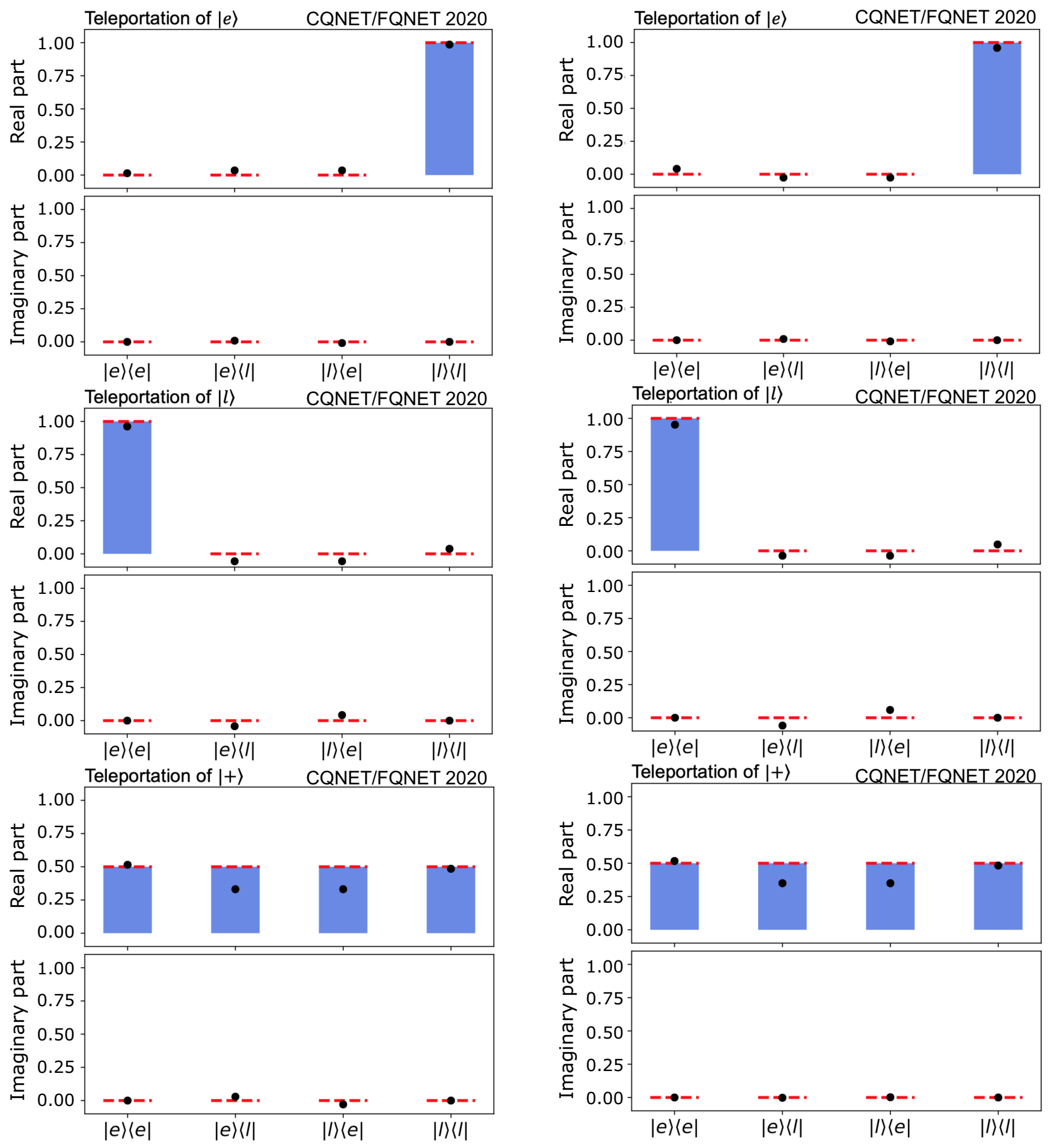

FIG. 8. The elements of the density matrices of teleported $|e\rangle$, $|\nmid\rangle$, and $|+\rangle$ states with the additional $44 \mathrm{~km}$ of fiber in the system. The black points are generated by our teleportation system and the blue bars with red dashed lines are the values assuming ideal teleportation.

formed by the Pauli matrices $\sigma_{x}, \sigma_{y}$, and $\sigma_{z}$, i.e., measuring photons at each of the arrival times after the MZI and at varying $\varphi$. These results allow reconstruction of the density

FIG. 9. The elements of the density matrices of teleported $|e\rangle$, $|1\rangle$, and $|+\rangle$ states. The black points are generated by our teleportation system and the blue bars with red dashed lines are the values assuming ideal teleportation.

matrix of each teleported state, both with and without the additional $44 \mathrm{~km}$ of fiber, using maximum-likelihood estimation [52]. Our resultant matrices clearly match the 
expected teleported state, with the calculated high teleportation fidelities in Sec. III C, up to the aforementioned effects due to multiple photons and distinguishability.

[1] C. H. Bennett, G. Brassard, C. Crépeau, R. Jozsa, A. Peres, and W. K. Wootters, Teleporting an unknown quantum state via dual classical and Einstein-Podolsky-Rosen channels, Phys. Rev. Lett. 70, 1895 (1993).

[2] D. Bouwmeester, J.-W. Pan, K. Mattle, M. Eibl, H. Weinfurter, and A. Zeilinger, Experimental quantum teleportation, Nature 390, 575 (1997).

[3] D. Boschi, S. Branca, F. De Martini, L. Hardy, and S. Popescu, Experimental Realization of Teleporting an Unknown Pure Quantum State via Dual Classical and Einstein-Podolsky-Rosen Channels, Phys. Rev. Lett. 80, 1121 (1998).

[4] A. Furusawa, J. L. Sørensen, S. L. Braunstein, C. A. Fuchs, H. J. Kimble, and E. S. Polzik, Unconditional Quantum Teleportation, Science 282, 706 (1998).

[5] B. Hensen, H. Bernien, A. E. Dréau, A. Reiserer, N. Kalb, M. S. Blok, J. Ruitenberg, R. F. L. Vermeulen, R. N. Schouten, C. Abellán, W. Amaya, V. Pruneri, M. W. Mitchell, M. Markham, D. J. Twitchen, D. Elkouss, S. Wehner, T. H. Taminiau, and R. Hanson, Loophole-free Bell inequality violation using electron spins separated by 1.3 kilometres, Nature 526, 682 (2015).

[6] L. K. Shalm et al., Strong Loophole-Free Test of Local Realism, Phys. Rev. Lett. 115, 250402 (2015).

[7] M. Giustina et al., Significant-Loophole-Free Test of Bell's Theorem with Entangled Photons, Phys. Rev. Lett. 115, 250401 (2015).

[8] W. Rosenfeld, D. Burchardt, R. Garthoff, K. Redeker, N. Ortegel, M. Rau, and H. Weinfurter, Event-Ready Bell Test Using Entangled Atoms Simultaneously Closing Detection and Locality Loopholes, Phys. Rev. Lett. 119, 010402 (2017).

[9] P. Gao, D. L. Jafferis, and A. C. Wall, Traversable wormholes via a double trace deformation, J. High Energy Phys. 2017, 151 (2017).

[10] B. Yoshida and N. Y. Yao, Disentangling Scrambling and Decoherence via Quantum Teleportation, Phys. Rev. X 9, 011006 (2019).

[11] K. A. Landsman, C. Figgatt, T. Schuster, N. M. Linke, B. Yoshida, N. Y. Yao, and C. Monroe, Verified quantum information scrambling, Nature 567, 61 (2019).

[12] S. Lloyd, L. Maccone, R. Garcia-Patron, V. Giovannetti, Y. Shikano, S. Pirandola, L. A. Rozema, A. Darabi, Y. Soudagar, L. K. Shalm, and A. M. Steinberg, Closed Timelike Curves via Postselection: Theory and Experimental Test of Consistency, Phys. Rev. Lett. 106, 040403 (2011).

[13] M. Aspelmeyer, T. J. Kippenberg, and F. Marquardt, Cavity optomechanics, Rev. Mod. Phys. 86, 1391 (2014).

[14] P.-Y. Hou, Y.-Y. Huang, X.-X. Yuan, X.-Y. Chang, C. Zu, L. He, and L.-M. Duan, Quantum teleportation from light beams to vibrational states of a macroscopic diamond, Nat. Commun. 7, 11736 (2016).

[15] M. A. Nielsen and I. L. Chuang, Quantum Computation and Quantum Information (Cambridge University Press, USA, 2011), 10th ed.
[16] T. D. Ladd, F. Jelezko, R. Laflamme, Y. Nakamura, C. Monroe, and J. L. O'Brien, Quantum computers, Nature 464, 45 (2010).

[17] N. Gisin and R. Thew, Quantum communication, Nat. Photonics 1, 165 (2007).

[18] S. Pirandola, J. Eisert, C. Weedbrook, A. Furusawa, and S. L. Braunstein, Advances in quantum teleportation, Nat. Photonics 9, 641 (2015).

[19] H.-J. Briegel, W. Dür, J. I. Cirac, and P. Zoller, Quantum Repeaters: The Role of Imperfect Local Operations in Quantum Communication, Phys. Rev. Lett. 81, 5932 (1998).

[20] H. J. Kimble, The quantum internet, Nature 453, 1023 (2008).

[21] C. Simon, Towards a global quantum network, Nat. Photonics 11, 678 (2017).

[22] S. Wehner, D. Elkouss, and R. Hanson, Quantum internet: A vision for the road ahead, Science 362, eaam9288 (2018).

[23] N. Sangouard, C. Simon, H. De Riedmatten, and N. Gisin, Quantum repeaters based on atomic ensembles and linear optics, Rev. Mod. Phys. 83, 33 (2011).

[24] J. Dias and T. C. Ralph, Quantum repeaters using continuous-variable teleportation, Phys. Rev. A 95, 022312 (2017).

[25] M. Lucamarini, Z. L. Yuan, J. F. Dynes, and A. J. Shields, Overcoming the rate-distance limit of quantum key distribution without quantum repeaters, Nature 557, 400 (2018).

[26] M. K. Bhaskar, R. Riedinger, B. Machielse, D. S. Levonian, C. T. Nguyen, E. N. Knall, H. Park, D. Englund, M. Lončar, D. D. Sukachev, and M. D. Lukin, Experimental demonstration of memory-enhanced quantum communication, Nature 580, 60 (2020).

[27] X.-X. Xia, Q.-C. Sun, Q. Zhang, and J.-W. Pan, Long distance quantum teleportation, Quantum Sci. Technol. 3, 014012 (2017).

[28] X.-S. Ma, T. Herbst, T. Scheidl, D. Wang, S. Kropatschek, W. Naylor, B. Wittmann, A. Mech, J. Kofler, E. Anisimova, et al., Quantum teleportation over 143 kilometres using active feed-forward, Nature 489, 269 (2012).

[29] T. Herbst, T. Scheidl, M. Fink, J. Handsteiner, B. Wittmann, R. Ursin, and A. Zeilinger, Teleportation of entanglement over $143 \mathrm{~km}$, Proc. Natl. Acad. Sci. 112, 14202 (2015).

[30] J. Yin, J.-G. Ren, H. Lu, Y. Cao, H.-L. Yong, Y.-P. Wu, C. Liu, S.-K. Liao, F. Zhou, Y. Jiang, et al., Quantum teleportation and entanglement distribution over 100-kilometre free-space channels, Nature 488, 185 (2012).

[31] J.-G. Ren, P. Xu, H.-L. Yong, L. Zhang, S.-K. Liao, J. Yin, W.-Y. Liu, W.-Q. Cai, M. Yang, L. Li, et al., Ground-tosatellite quantum teleportation, Nature 549, 70 (2017).

[32] J. Brendel, N. Gisin, W. Tittel, and H. Zbinden, Pulsed Energy-Time Entangled Twin-Photon Source for Quantum Communication, Phys. Rev. Lett. 82, 2594 (1999).

[33] R. Valivarthi, M. G. Puigibert, Q. Zhou, G. H. Aguilar, V. B. Verma, F. Marsili, M. D. Shaw, S. W. Nam, D. Oblak, and W. Tittel, Quantum teleportation across a metropolitan fibre network, Nat. Photonics 10, 676 (2016).

[34] Q.-C. Sun, Y.-L. Mao, S.-J. Chen, W. Zhang, Y.-F. Jiang, Y.-B. Zhang, W.-J. Zhang, S. Miki, T. Yamashita, H. Terai, X. Jiang, T.-Y. Chen, L.-X. You, X.-F. Chen, Z. Wang, J.-Y. Fan, Q. Zhang, and J.-W. Pan, Quantum teleportation with 
independent sources and prior entanglement distribution over a network, Nat. Photonics 10, 671 (2016).

[35] H. Takesue and B. Miquel, Entanglement swapping using telecom-band photons generated in fibers, Opt. Express 17, 10748 (2009).

[36] S.-K. Liao, Long- distance free-space quantum key distribution in daylight towards inter-satellite communication, Nat. Photonics 11, 509 (2017).

[37] A. I. Lvovsky, B. C. Sanders, and W. Tittel, Optical quantum memory, Nat. Photonics 3, 706 (2009).

[38] N. Lauk, N. Sinclair, S. Barzanjeh, J. P. Covey, M. Saffman, M. Spiropulu, and C. Simon, Perspectives on quantum transduction, Quantum Sci. Technol. 5, 020501 (2020).

[39] N. J. Lambert, A. Rueda, F. Sedlmeir, and H. G. Schwefel, Coherent Conversion Between Microwave and Optical Photons-An Overview of Physical Implementations, Adv. Quantum Technol. 3, 1900077 (2020).

[40] V. B. Braginsky and F. Y. Khalili, Quantum non-demolition measurements: the route from toys to tools, Rev. Mod. Phys. 68, 1 (1996).

[41] I. Marcikic, H. De Riedmatten, W. Tittel, H. Zbinden, and N. Gisin, Long-distance teleportation of qubits at telecommunication wavelengths, Nature 421, 509 (2003).

[42] H. De Riedmatten, I. Marcikic, W. Tittel, H. Zbinden, D. Collins, and N. Gisin, Long Distance Quantum Teleportation in a Quantum Relay Configuration, Phys. Rev. Lett. 92, 047904 (2004).

[43] H. Takesue, S. D. Dyer, M. J. Stevens, V. Verma, R. P. Mirin, and S. W. Nam, Quantum teleportation over $100 \mathrm{~km}$ of fiber using highly efficient superconducting nanowire single-photon detectors, Optica 2, 832 (2015).

[44] O. Landry, J. A. W. van Houwelingen, A. Beveratos, H. Zbinden, and N. Gisin, Quantum teleportation over the Swisscom telecommunication network, JOSA B 24, 398 (2007).

[45] M. Halder, A. Beveratos, N. Gisin, V. Scarani, C. Simon, and $\mathrm{H}$. Zbinden, Entangling independent photons by time measurement, Nat. Phys. 3, 692 (2007).

[46] F. Bussières, C. Clausen, A. Tiranov, B. Korzh, V. B. Verma, S. W. Nam, F. Marsili, A. Ferrier, P. Goldner, H. Herrmann, C. Silberhorn, W. Sohler, M. Afzelius, and N. Gisin, Quantum teleportation from a telecom- wavelength photon to a solid-state quantum memory, Nat. Photonics 8 , 775 (2014).

[47] P. Gao and D. L. Jafferis, A Traversable Wormhole Teleportation Protocol in the SYK Model, arXiv:1911.07416 [hep-th] (2019).

[48] E. Miyazono, T. Zhong, I. Craiciu, J. M. Kindem, and A. Faraon, Coupling of erbium dopants to yttrium orthosilicate photonic crystal cavities for on-chip optical quantum memories, Appl. Phys. Lett. 108, 011111 (2016).

[49] B. Lauritzen, J. Mináŕ, H. De Riedmatten, M. Afzelius, N. Sangouard, C. Simon, and N. Gisin, TelecommunicationWavelength Solid-State Memory at the Single Photon Level, Phys. Rev. Lett. 104, 080502 (2010).

[50] S. Welinski, P. J. T. Woodburn, N. Lauk, R. L. Cone, C. Simon, P. Goldner, and C. W. Thiel, Electron Spin Coherence in Optically Excited States of Rare-Earth Ions for Microwave to Optical Quantum Transducers, Phys. Rev. Lett. 122, 247401 (2019).
[51] C. K. Hong, Z. Y. Ou, and L. Mandel, Measurement of subpicosecond time intervals between two photons by interference, Phys. Rev. Lett. 59, 2044 (1987).

[52] J. B. Altepeter, E. R. Jeffrey, and P. G. Kwiat, Photonic State Tomography, Adv. At., Mol., Opt. Phys. 52, 105 (2005).

[53] X. Ma, B. Qi, Y. Zhao, and H.-K. Lo, Practical decoy state for quantum key distribution, Phys. Rev. A 72, 012326 (2005).

[54] G. Iskander, N. Sinclair, C. Peña, S. Xie, and M. Spiropulu, Stabilization of an electro-optic modulator for quantum communication using a low-cost microcontroller, Caltech Undergraduate Res. J. 20 (2019).

[55] J. Rarity, Interference of Single Photons from Separate Sources, Ann. N. Y. Acad. Sci. 755, 624 (1995).

[56] F. Marsili, V. B. Verma, J. A. Stern, S. Harrington, A. E. Lita, T. Gerrits, I. Vayshenker, B. Baek, M. D. Shaw, R. P. Mirin, and S. W. Nam, Detecting single infrared photons with 93\% system efficiency, Nat. Photonics 7, 210 (2013).

[57] Photon spot, Sub-Kelvin Cryogenics \& Superconducting Nanowire Single-Photon Detectors, https://www.photon spot.com/.

[58] N. Lütkenhaus, J. Calsamiglia, and K.-A. Suominen, Bell measurements for teleportation, Phys. Rev. A 59, 3295 (1999).

[59] I. Marcikic, H. de Riedmatten, W. Tittel, V. Scarani, H. Zbinden, and N. Gisin, Time-bin entangled qubits for quantum communication created by femtosecond pulses, Phys. Rev. A 66, 062308 (2002).

[60] L. Mandel and E. Wolf, Optical Coherence and Quantum Optics, Optical Coherence and Quantum Optics (Cambridge University Press, Cambridge, 1995).

[61] T. Zhong and F. N. Wong, Nonlocal cancellation of dispersion in Franson interferometry, Phys. Rev. A 88, 020103 (2013).

[62] Manuscript in preparation.

[63] R. F. Werner, Quantum states with Einstein-PodolskyRosen correlations admitting a hidden-variable model, Phys. Rev. A 40, 4277 (1989).

[64] J. F. Clauser, M. A. Horne, A. Shimony, and R. A. Holt, Proposed Experiment to Test Local Hidden-Variable Theories, Phys. Rev. Lett. 23, 880 (1969).

[65] H. Takesue, $1.5 \mu \mathrm{m}$ band Hong-Ou-Mandel experiment using photon pairs generated in two independent dispersion shifted fibers, Appl. Phys. Lett. 90, 204101 (2007).

[66] S. Massar and S. Popescu, Optimal Extraction of Information from Finite Quantum Ensembles, Phys. Rev. Lett. 74, 1259 (1995).

[67] H. P. Specht, C. Nölleke, A. Reiserer, M. Uphoff, E. Figueroa, S. Ritter, and G. Rempe, A single-atom quantum memory, Nature 473, 190 (2011).

[68] N. Sinclair, E. Saglamyurek, H. Mallahzadeh, J. A. Slater, M. George, R. Ricken, M. P. Hedges, D. Oblak, C. Simon, W. Sohler, and W. Tittel, Spectral Multiplexing for Scalable Quantum Photonics using an Atomic Frequency Comb Quantum Memory and Feed-Forward Control, Phys. Rev. Lett. 113, 053603 (2014).

[69] D. Bruss, A. Ekert, and C. Macchiavello, Optimal Universal Quantum Cloning and State Estimation, Phys. Rev. Lett. 81, 2598 (1998). 
[70] V. Bužek and M. Hillery, Quantum copying: Beyond the no-cloning theorem, Phys. Rev. A 54, 1844 (1996).

[71] M. Takeoka, R.-B. Jin, and M. Sasaki, Full analysis of multi-photon pair effects in spontaneous parametric down conversion based photonic quantum information processing, New J. Phys. 17, 043030 (2015).

[72] C. Weedbrook, S. Pirandola, R. García-Patrón, N. J. Cerf, T. C. Ralph, J. H. Shapiro, and S. Lloyd, Gaussian quantum information, Rev. Mod. Phys. 84, 621 (2012).

[73] D. Zhu, M. Colangelo, C. Chen, B. A. Korzh, F. N. Wong, M. D. Shaw, and K. K. Berggren, Resolving Photon Numbers Using a Superconducting Nanowire with ImpedanceMatching Taper, Nano Lett. 20, 3858 (2020).

[74] H. Krovi, S. Guha, Z. Dutton, J. A. Slater, C. Simon, and W. Tittel, Practical quantum repeaters with parametric down-conversion sources, Appl. Phys. B 122, 52 (2016).

[75] P. J. Mosley, J. S. Lundeen, B. J. Smith, P. Wasylczyk, A. B. U'Ren, C. Silberhorn, and I. A. Walmsley, Heralded Generation of Ultrafast Single Photons in Pure Quantum States, Phys. Rev. Lett. 100, 133601 (2008).

[76] R. M. Rassoul, A. Ivanov, E. Freysz, A. Ducasse, and F. Hache, Second-harmonic generation under phase- velocity and group-velocity mismatch: influence of cascading self-phase and cross-phase modulation, Opt. Lett. 22, 268 (1997).

[77] B. Korzh, Q.-Y. Zhao, J. P. Allmaras, S. Frasca, T. M. Autry, E. A. Bersin, A. D. Beyer, R. M. Briggs, B. Bumble, M. Colangelo, et al., Demonstration of sub-3 ps temporal resolution with a superconducting nanowire single-photon detector, Nat. Photonics 14, 250 (2020).

[78] R. Valivarthi, I. Lucio-Martinez, A. Rubenok, P. Chan, F. Marsili, V. B. Verma, M. D. Shaw, J. A. Stern, J. A. Slater, D. Oblak, S. W. Nam, and W. Tittel, Efficient Bell state analyzer for time-bin qubits with fast-recovery WSi superconducting single photon detectors, Opt. Express 22, 24497 (2014).

[79] R. Valivarthi, Q. Zhou, C. John, F. Marsili, V. B. Verma, M. D. Shaw, S. W. Nam, D. Oblak, and W. Tittel, A costeffective measurement-device-independent quantum key distribution system for quantum networks, Quantum Sci. Technol. 2, 04LT01 (2017).

[80] Q.-C. Sun, Y.-L. Mao, Y.-F. Jiang, Q. Zhao, S.-J. Chen, W. Zhang, W.-J. Zhang, X. Jiang, T.-Y. Chen, L.-X. You, L. Li, Y.-D. Huang, X.-F. Chen, Z. Wang, X. Ma, Q. Zhang, and J.-W. Pan, Entanglement swapping with independent sources over an optical-fiber network, Phys. Rev. A 95, 032306 (2017).

[81] S. L. Braunstein and S. Pirandola, Side-Channel-Free Quantum Key Distribution, Phys. Rev. Lett. 108, 130502 (2012).

[82] D. Gottesman and I. L. Chuang, Demonstrating the viability of universal quantum computation using teleportation and single-qubit operations, Nature 402, 390 (1999).

Correction: An Award number was missing in the Acknowledgments section and has been inserted. 